\title{
Murine pro-tumor necrosis factor expressed in Saccharomyces cerevisiae HF7c localizes to membrane/particulate
}

\author{
Jae-Yeon Jeong ${ }^{1}$ and Dae-Myung Jue ${ }^{1,2}$ \\ ${ }^{1}$ Department of Biochemistry, College of Medicine, \\ The Catholic University of Korea, 505 Banpo-Dong, \\ Socho-Ku, Seoul 137-701, Korea \\ Corresponding author: Tel, +82-2-590-1177; \\ Fax, +82-2-596-4435; E-mail, dmjue@cmc.cuk.ac.kr
}

Accepted 18 May 2000

Abbreviations: TNF, tumor necrosis factor; TACE, TNF- $\alpha$ converting enzyme

\begin{abstract}
Tumor necrosis factor (TNF) is a cytokine that is produced by immune cells in response to bacterial and viral stimuli and plays important roles in various inflammatory diseases. TNF is produced as a membrane-bound precursor, which is then cleaved to release soluble mature protein. We expressed murine pro-TNF in Saccharomyces cerevisiae and examined processing and cellular localization of the recombinant protein. Yeast cells were transformed with an expression construct carrying the pro-TNF gene under the control of alcohol dehydrogenase promoter. Immunoblotting analysis of cell homogenate revealed expression of $\mathbf{2 6} \mathrm{kD}$ pro-TNF in transformed cells. Upon centrifugation, pro-TNF transformed cells fractionated into the membrane/particulate. In a clone that expresses a high level of pro-TNF, mature $17 \mathrm{kD}$ TNF was detected in the culture medium, although the amount was far smaller than that of cell-associated pro-TNF. Flow cytometric analysis of yeast spheroplasts demonstrated the presence of TNF on the cell surface. Our results show that proTNF expressed in yeast mainly resides in the cellular membrane with an orientation similar to that of proTNF produced in mammalian cells. Our data suggest that the transformed yeast cells can be used for the genetic analysis of pro-TNF processing machinery in immune cells.
\end{abstract}

Keywords: Tumor necrosis factor, protein processing, post-translational, Saccharomyces cerevisiae, cell surface proteins, membrane proteins

\section{Introduction}

Tumor necrosis factor (TNF, also called TNF- $\alpha$ ) is a cytokine that has been implicated as an important mediator of shock and tissue injury provoked by bacterial endotoxin (Beutler et al., 1985; Tracey et al., 1986). It also plays roles in development and maintenance of chronic inflammatory diseases such as rheumatoid arthritis, autoimmune diabetes, Crohns disease and inflammatory bowl disease (Vassalli, 1992; Tracey and Cerami, 1993; van Dullemen et al., 1995). Initially discovered as an immune mediator that induces hemorrhagic necrosis of tumors in experimental animals and death of cultured tumor cells, TNF regulates expression of stress response genes by activating kinase cascades on the one side and target cell survival and death by activating death domain proteins on the other (Wallach et al., 1999). TNF is mainly produced by monocytes/macrophages in response to microbial and parasitic stimuli, but other cells including, lymphocytes, natural killer cells, Kupffer cells, glial cells, and adipocytes also produce TNF.

Human TNF is initially synthesized as a membranebound 233 amino acid (235 amino acid in mouse) precursors with $\mathrm{N}$-terminal propeptides of 76 amino acids (79 amino acids in mouse) containing a hydrophobic membrane-anchoring sequence. The $26 \mathrm{kD}$ pro-TNF is proteolytically cleaved to release soluble mature $17 \mathrm{kD}$ protein into the medium, and the prosequence with apparent mass of $14 \mathrm{kD}$ remains cell associated (Kriegler et al., 1988; Jue et al., 1990). Processing of pro-TNF was shown to be blocked by inhibitors of metalloproteinases and serine proteinases, suggesting that one or more of these proteolytic enzymes is involved in cleavage and shedding (Kim et al.,1993; Gearing et al., 1994; McGeehan et al., 1994; Mohler et al., 1994). In subsequent studies a TNF- $\alpha$ converting enzyme (TACE) has been identified and characterized as a membraneanchored metalloproteinase that is a member of ADAM (a disintegrin and metalloproteinase) domain family that combines features of both cell adhesion molecules and proteolytic enzymes (Black et al., 1997; Moss et al., 1997). Its role in pro-TNF processing has been supported by studies using mutant mice that are deficient in the TACE activity (Black et al., 1997). T-cells derived from these mice showed an $80-90 \%$ reduction in TNF release and an increase in surface expression of pro-TNF compared with normal cells. However, the residual 10-20\% release of TNF was still detected in the mutant cells, and this was not blocked by a metalloproteinase inhibitor (Black et al., 1997). Moreover, expression of a mutant 
form of TACE in normal cells was shown to inhibit endogenous TACE function, indicating that the reduced TNF release in mutant $T$ cells was due to a dominant negative effect of mutant TACE rather than the absence of other TACE-like enzymes (Solomon et al., 1999). Recent reports also implicated another ADAM family proteinase ADAM 10 as a TNF convertase (Lunn et al., 1997; Rosendahl et al., 1997). These results suggest that alternative pathways for the release of membranebound pro-TNF exist in certain types of cells. In this study, we expressed murine pro-TNF in yeast and examined processing and cellular localization of the recombinant protein in transformed cells. TNF was not actively secreted in yeast cells and largely remained in the membrane/particulate fraction, indicating that these cells can be used for the characterization of mammalian genes involved in the processing of TNF.

\section{Materials and Methods}

\section{Materials}

Saccharomyces cerevisiae strain HF7c (MATa, his 3200, leu 2-3, trp 1-901, ura3-52) and plasmid pGBT9 were components of a yeast two-hybrid system and obtained from Clontech (Palo Alto, CA). HF7c strain has trp1 and leu2 as auxotrophic markers and cannot grow in minimal medium lacking tryptophan and leucine. RAW 264.7 murine macrophages were obtained from American Type Culture Collection (Rockville, MD) and maintained as described previously (Jeong and Jue, 1997). Recombinant murine TNF was expressed in E. coli and purified as described previously (Jeong and Jue, 1997). Polyclonal antibody to murine TNF was obtained by immunizing rabbits with recombinant protein.

\section{Plasmid construction}

Full-length pro-TNF cDNA was amplified by reverse transcription (RT)-PCR from RNA purified from lipopolysaccharide-treated RAW 264.7 cells and cloned into the yeast expression vector pGBT9. The $5^{\prime}$ primer (CGCAAGCTTATGAGCACAGAAAGC) included an HindllI site, and the 3' primer (CGGAATTCCTCACAGAGCAATGAC) included an EcoRI site for cloning. pGBT9 is constructed to generate a fusion protein of the target with the GAL4 DNA-binding domain which resides between the yeast alcohol dehydrogenase promoter and the multiple cloning site. To remove GAL4 DNA-binding domain, pGBT9 is completely digested with EcoRI, and then partially digested with HindIII. The reaction products were separated by electrophoresis on a $0.5 \%$ agarose gel and an $5.1 \mathrm{~kb}$ fragment devoid of the GAL4 DNA-binding domain (pGBT9 $\Delta$ ) was isolated and ligated to pro-TNF cDNA digested with HindIII and EcoRI. Bacterial colonies were screened by PCR to select transformants. Recombinant expression vector (pGBT9 $\Delta$ proTNF) isolated from tranformed $E$. coli cells was analyzed by restriction enzyme digestion and DNA sequencing.

\section{Transformation and culture of yeast cells}

Preparation of competent yeast cells and transformation with pGBT9 $\Delta$-proTNF were carried out according to the protocol provided by Clontech. The competent cells were prepared by lithium acetate method and transformed with $0.1 \mu \mathrm{g}$ of pGBT9 $\Delta$-proTNF. The transformed cells were plated onto $10-\mathrm{cm}$ dishes containing minimal SD agar in which tryptophan was omitted (SD-Trp) and were allowed to grow for 3 days at $30^{\circ} \mathrm{C}$. To determine if the transformants harbor pro-TNF expression plasmids, colonies grown on the selective medium were used to inoculate $2 \mathrm{ml}$ of YPD medium and cultured at $30^{\circ} \mathrm{C}$ overnight with shaking. DNA was isolated from the yeast cells by disrupting the cells with glass beads in chloroform/phenol mixture (Hoffman and Winston, 1987). The isolated DNA contained chromosomal as well as plasmid DNA, and the presence of pGBT9 was determined by PCR using primers that amplify proTNF cDNA.

\section{Analysis of TNF in yeast homogenate and culture supernatant}

Wild-type HF7c and transformed yeast clones were cultured in $2 \mathrm{ml}$ of YPD medium for 2 days and protein extracts were prepared using glass beads as described previously (Dunn and Wobbe, 1993). Equal volumes (7 $\mu \mathrm{l})$ of yeast homogenate were analyzed by SDS-PAGE on a $15 \%$ gel along with prestained molecular weight standards (Bio-Rad, Hercules, CA). The proteins were transferred to a nitrocellulose membrane, and pro-TNF was visualized with anti-TNF antibody using an ECL detection kit (Amersham, Buckinghamshire, UK) (Yang et al., 1999). To determine subcellular localization of TNF-related proteins, the yeast crude homogenate was centrifuged for $20 \mathrm{~min}$ at $12,000 \mathrm{~g}$ and the supernatant (cytosol) and the precipitate (membrane/particulate) fractions were analyzed by immunoblotting along with culture supernatant. TNF released in the yeast culture supernatant was also measured by L-929 cell cytotoxicity assay after the sample was dialyzed against PBS overnight in a microdialyzer (Pierce, Rockford, IL) (Jue et al., 1990).

\section{Flow cytometry of cell surface TNF}

Yeast cells grown in YPD medium overnight were harvested by centrifugation at $1,500 \mathrm{~g}$ for $5 \mathrm{~min}$. Cells of 3 $\mu$ l packed volume were fixed in $4 \%$ paraformaldehyde, and the cell wall was removed by incubating the cells with $\beta$-glucuronidase (1000 U, Sigma, St. Louis, MO) and lyticase (12 $\mu \mathrm{g}$, Sigma) in $100 \mu \mathrm{l}$ of sorbitol buffer 
(Kilmartin and Adams, 1984). The yeast spheroplasts were incubated with $1 \mu \mathrm{g}$ of rat anti-mouse TNF monoclonal antibody (Pharmingen, San Diego, CA) for 30 min on ice. After washing, the cells were incubated with fluorescein isothiocyanate-labeled goat anti-rat IgG (Sigma) for $30 \mathrm{~min}$ on ice and the expression of cell surface TNF was analyzed on a flow cytometer (FACSorter, Becton-Dickinson, Moutain View, CA).

\section{Results and Discussion}

In this study, we sought to establish an yeast cell line which expresses pro-TNF and can be used for the genetic analysis of pro-TNF processing machinery in mammalian cells. For the study, we used $S$. cerevisiae strain HF7c, because it has two auxotrophic markers, trp1 and leu2, and thus can be transfected simultaneously with two different expression vectors, i.e. one encoding pro-TNF and the other encoding candidate mammalian genes. The yeast cells were initially transformed with pGBT9D-proTNF containing leu2 selection marker and murine pro-TNF gene under the control of yeast alcohol dehydrogenase promoter. The original pGBT9 was constructed to express target protein as a fusion protein with GAL4 DNA-binding domain. After synthesis, the expressed fusion protein translocates to the nucleus because of its nuclear localization signal in the DNA-binding domain. We thus removed the coding region for GAL4 DNA-binding domain from pGBT9 using HindIII and EcoRI sites flanking the region and inserted pro-TNF cDNA instead. Restriction enzyme analysis and DNA sequencing of obtained recombinant construct indicated that pro-TNF cDNA was ligated to pGBT9D in an expected direction.

The prepared pro-TNF expression vector was then used to transfect yeast cells and transformants were grown on selective agar plates containing leucine but lacking tryptophan. The number of colonies obtained from transformation with $0.1 \mu \mathrm{g}$ of pGBT9D-proTNF was about $1 \times 10^{4}$. To test whether the transformants produce TNF, colonies were randomly selected, and cultured in YPD medium, and TNF associated with the cell and released in culture supernatant was measured by immunoblotting analysis. Our result showed that most clones did not release detectable amounts of TNF in the culture supernatant. In the crude homogenate of certain yeast cells, however, significant level of $26 \mathrm{kD}$ protein reacting with anti-TNF antibody was detected, indicating that pro-TNF produced by the transformants is largely associated with the cell (Figure 1). The $26 \mathrm{kD}$ protein was not produced from untransfected HF7c cells, further supporting our argument that the detected protein derived from the transfected pro-TNF gene. The expression levels of pro-TNF were diverse among different clones due to an unknown reason. However, in some clones

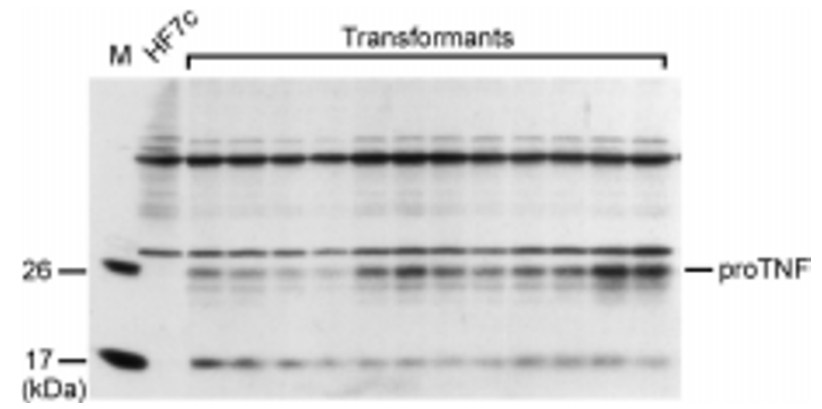

Figure 1. Immunoblotting analysis of pro-TNF produced in yeast transformants. Clones of $S$. cerevisiae HF7c cells transformed with pGBT9AproTNF (Transformants) and untransformed HF7c cells were cultured in YPD medium for 2 days. Crude homogenate was prepared from yeast pellet by glass disruption and analyzed by immunoblotting analysis using anti-TNF antibody. Lane marked $\mathrm{M}$ shows recombinant glutathione S-transferase and mature TNF, which have molecular masses of $26 \mathrm{kD}$ and $17 \mathrm{kD}$, respectively, used as molecular weight markers. The result is a representative of five similar experiments with different clones.

the level was correlated with that of pGBT9 $\Delta$-proTNF detected in yeast cells (see below). The different expression levels were maintained throughout successive cultures and after reviving of frozen stock culture, indicating that the trait is stable and not due to variation in the culture conditions.

Based on the result of immunoblotting analysis, we selected two yeast clones that produce either low or high levels of pro-TNF for further characterizations. To test whether their ability to produce pro-TNF is correlated with the level of expression plasmids in the cell, yeast DNA was isolated from the transformants and parental HF7c cells, and the level of pGBT9 $\Delta$-proTNF was determined by PCR. As shown in Figure $2 A$, the control reaction performed with purified pGBT9s-proTNF as a template gave a major amplified product of expected 726-bp size. A comparable level of PCR product was detected in the reaction performed with DNA from high-producer yeast clone, while reaction with DNA from low-producer clone gave significantly lower level of PCR product. Amplified DNA was not detected in a reaction carried out with DNA obtained from untransformed HF7c cells.

Our result demonstrates a direct association of the cellular pGBT9 expression in low and high producer yeast clones.

Pro-TNF produced in mammalian cells has been shown to localize to membrane/particulate fraction through its membrane anchoring sequence in the prosequence (Kriegler et al., 1988; Jue et al., 1990). To examine the cellular localization of pro-TNF in yeast, transformants and parental HF7c cells were homogenized by glass bead disruption, and the cytosolic and membrane/particulate fractions were isolated after centrifugation. Immunoblotting analysis of TNF in each fractions revealed that pro-TNF also localizes to membrane/particulate in yeast cells (Figure 2B). In the high producer clone, most 


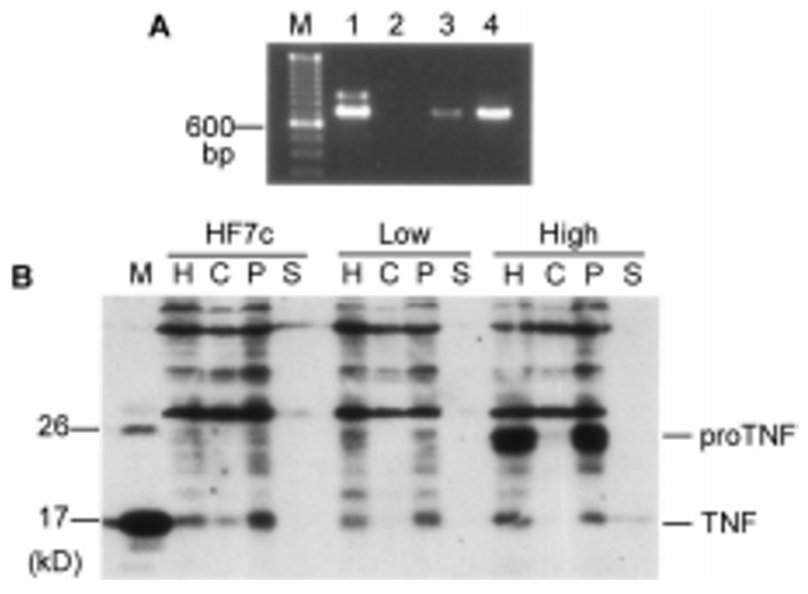

Figure 2. Plasmid DNA and pro-TNF levels in low and high producer clones and subcellular localization of pro-TNF. A. Yeast DNA was isolated from parental HF7c cells (lane 2), and transformed yeast cells producing low (lane 3), or high (lane 4) level of pro-TNF. The level of pGBT9 $\Delta$-proTNF was measured by PCR using primers that amplify 726-bp pro-TNF cDNA. Lane $M$ shows 100-bp ladder DNA marker. PCR product obtained from pGBT9 $\Delta$ proTNF is shown in lane 1 as a control. B. Yeast clones were cultured in YPD medium and homogenate $(\mathrm{H})$ was prepared. After centrifugation at $12,000 \mathrm{~g}$ for $20 \mathrm{~min}$, the supernatant (cytosol, C) and pellet (membrane/ particulate, $\mathrm{P}$ ) were obtained and the pellet was resuspended in the same volume of cell disruption buffer. Proteins in culture supernatant (S) were precipitated with 2 volumes of cold acetone and resuspended in $2 \times$ SDSPAGE sample buffer. For comparison, equal portions of cellular fractions and culture supernatant was analyzed by immunoblotting using anti-TNF antibody. Lane $\mathrm{M}$ shows recombinant glutathione S-transferase and mature TNF as molecular weight markers. The blot was overexposed to reveal mature TNF in the culture supernatant of high producer clone.

of the pro-TNF detected in homogenate was fractionated into membrane/particulate after centrifugation and none could be detected in the cytosolic fraction. The amount of pro-TNF in low producer cells was relatively small compared with that of high producer cells.

In some clones that produce relatively large amounts of pro-TNF, a band migrating to $17 \mathrm{kD}$ position was also detected in crude homogenates by immunoblotting analysis (Figure 1). Although the amount of $17 \mathrm{kD}$ protein was relatively small compared with that of pro-TNF in the same cells, this result suggests that yeast cells can process pro-TNF to make mature protein in a way similar to mammalian cells. A small but detectable amount of $17 \mathrm{kD}$ TNF-like protein was detected also in the culture supernatant of high producer yeast clone, suggesting that the processed mature TNF is released in the medium (Figure 2B, lane 13). To examine whether mature TNF is indeed secreted from yeast cells transformed with pGBT9 $\Delta$-proTNF, TNF activity in the yeast culture supernatant was measured by determining its cytotoxicity to TNF-sensitive L-929 cells. Our result shown in Figure 3 indicated that the culture supernatant of high producer clone indeed induced death of target cells at low dilutions. These results provide evidences that proTNF is processed in yeast cells to $17 \mathrm{kD}$ mature protein,

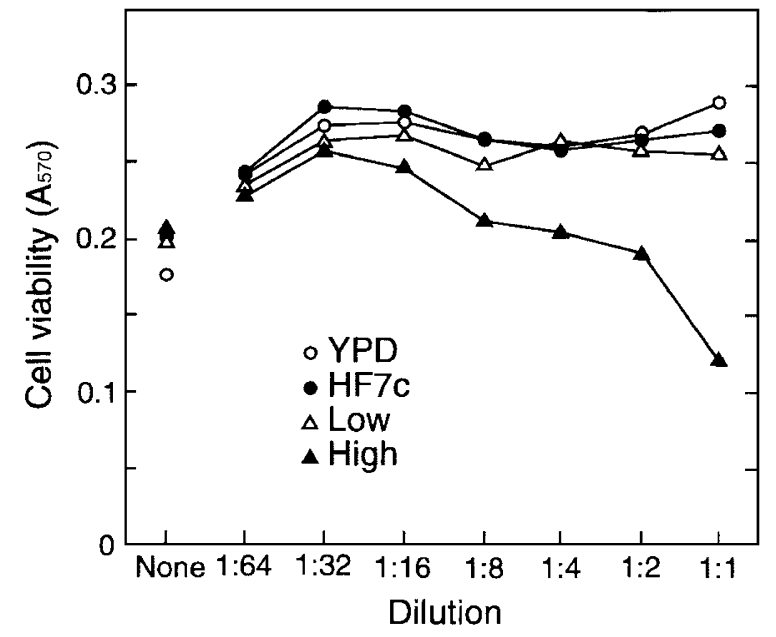

Figure 3. Cytotoxic activity of yeast culture supernatants. Parental HF7c and low and high producer yeast transformants were grown in YPD medium for $24 \mathrm{~h}$. The culture supernatant was dialyzed against PBS and $10 \mu \mathrm{l}$ aliquots were added to L-929 cells in 2-fold serial dilutions. After incubation for $16 \mathrm{~h}$, viable cells were stained with 3-(4,5-dimethylthiazol-2-yl)-2,5diphenyltetrazolium bromide (MTT) and absorbance was measured at 570 $\mathrm{nm}$. YPD medium dialyzed against PBS was tested as a blank.

and a certain portion of it is secreted like in mammalian cells. However, densitometric measurement of immunoblot revealed that in yeast cells the level of mature TNF in the medium after 24 -h culture was only $3 \%$ of cellassociated pro-TNF (Figure 2B), indicating most of TNF produced remained in the cell as a proprotein. On the contrary, $96 \%$ of TNF produced from RAW 264.7 murine macrophages were released in the culture medium as mature protein, while only $4 \%$ remained in the cell after $16 \mathrm{~h}$ of LPS stimulation (Jue et al., 1990). Therefore, although a portion of overexpressed pro-TNF is converted to mature form in yeast cells, the efficiency seems to be far lower than that of mammalian cells.

In mammalian cells pro-TNF has been shown to be synthesized as a type II membrane protein, with its Cterminus containing mature protein exposed to the luminal side of endoplasmic reticulum (thus extracellular side of plasma membrane), and its N-terminus to cytosolic side (Kriegler et al., 1988; Hofsli et al., 1989). We examined the orientation of pro-TNF produced in yeast cells by staining yeast spheroplasts sequentially with anti-TNF antibody and fluorescence-labeled secondary antibody and analyzing them on a flow cytometer. To detect cell surface TNF, the spheroplasts were not permeabilized and the antibody binding reaction was performed at low temperature. As shown in Figure 4, the signal for TNF on the surface of transformed high producer yeast cells was significantly elevated compared with that of untransformed HF7c cells. Low producer yeast clone displayed an intermediate level of signal between those of the high producer cells and HF7c cells (data not shown). Our result indicated that, like in mammalian cells, pro-TNF synthesized in yeasts cells is 


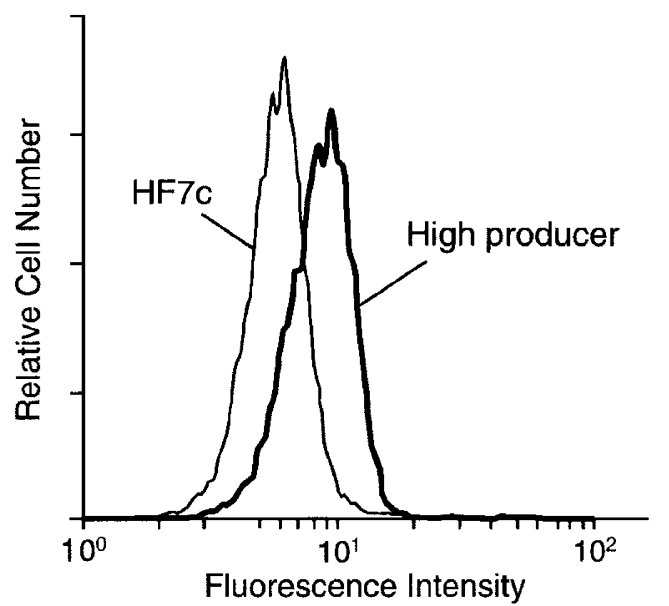

Figure 4. Flow cytometric measurement of cell surface TNF. HF7c cells and high producer pGBT9 $\Delta$-proTNF transformant were grown in YPD medium overnight. The cells were fixed and the cell wall was digested with $\beta$-glucuronidase and lyticase. The spheroplasts were sequentially incubated with anti-murine TNF antibody and fluorescein isothiocyanate-labeled secondary antibody at low temperature and analyzed by flow cytometry.

expressed on the cell surface with its C-terminal portion containing mature protein facing extracellular space.

In summary, these results show that like in mammalian cells murine pro-TNF expressed in yeast cells is mainly localized to membrane/particulate, while processing of this proprotein to mature form in yeast cells is minimal compared with that in mammalian cells. Flow cytometric analysis revealed that orientation of expressed protein on the plasma membrane is similar to that of mammalian cells. Our findings indicate that these yeast clones provide an appropriate tool for genetic characterization of pathways involved in processing and secretion of pro-TNF in mammalian cells. In a previous study with null mutant mice, TACE was also shown to play essential roles in cell surface shedding of TNF receptor p75, cell adhesion molecule L-selectin, and transforming growth factor $\alpha$, an epidermal growth factor ligand (Peschon et al., 1998). TACE has also been implicated in the cleavage of amyloid protein precursor, a membrane-bound precursor $A \beta$ peptide that accumulates in the brain of Alzheimer patients, within the $A \beta$ domain ( $\alpha$ cleavage), thus contributing to reduced formation of $A \beta$ peptide (Buxbaum et al., 1998; Merlos-Suarez et al., 1998). Many other proteins and receptors are known to shed on the cell surface by proteolysis, although enzymes involved in their processing largely remain elusive (Werb and Yan, 1998). By using our method, it should be possible to characterize the proteolytic enzymes involved and their control mechanisms.

\section{Acknowledgement}

This work was supported by a grant from the Korea
Science and Engineering Foundation (Grant No. KOSEF 951-0100-001-2).

\section{References}

Black, R. A., Rauch, C. T., Koziosky, C. J., Peschon, J. J., Slack, J. L., Wolfson, M. F., Castner, B. J., Stocking, K. L., Reddy, P., Srinivasan, S., Nelson, N., Boiani, N., Schooley, K. A., Gerhart, M., Davis, R., Fitzner, J. N., Johnson, R. S., Paxton, R. J., March, C. J. and Cerretti, D. P. (1997) A metalloproteinase disintegrin that releases tumour-necrosis factor- $\alpha$ from cells. Nature 385: 729-733

Beutler, B., Milsark, I. W. and Cerami, A. C. (1985) Passive immunization against cachectin/tumor necrosis factor protects mice from lethal effect of endotoxin. Science 229: 869-871

Buxbaum, J. D., Liu, K. N., Luo, Y., Slack, J. L., Stocking, K. L., Peschon, J. J., Johnson, R. S., Castner, B. J., Cerretti, D. P. and Black, R. A. (1998) Evidence that tumor necrosis factor $\alpha$ converting enzyme is involved in regulated alpha-secretase cleavage of the Alzheimer amyloid protein precursor. J. Biol. Chem. 273: 27765-27767

van Dullemen, H. M., van Deventer, S. J., Hommes, D. W., Bijl, H. A., Jansen, J., Tytgat, G. N. and Woody, J. (1995) Treatment of Crohn's disease with anti-tumor necrosis factor chimeric monoclonal antibody (cA2). Gastroenterology 109: 129-135

Dunn, B. and Wobbe, C. R. (1993) Preparation of protein extracts from yeast. In Current Protocols in Molecular Biology (Ausbel, F. M., Brent, R., Kingston, R. E., Moore, D. D., Seidman, J. G., Smith, J. A. and Struhl, K., eds.), p.13.13, Wiley Interscience, Brooklyn, NY

Gearing, A. J., Beckett, P., Christodoulou, M., Churchill, M., Clements, J., Davidson, A. H., Drummond, A. H., Galloway, W. A., Gilbert, R., Gordon, J. L., Leber, T. M., Mangan, M., Miller, K., Nayee, P., Owen, K., Patel, S., Thomas, W., Wells, G., Wood, L. M. and Woolley, K. (1994) Processing of tumour necrosis factor- $\alpha$ precursor by metalloproteinases. Nature 370: 555-557

Hoffman, C. S. and Winston, F. (1987) A ten-minute DNA preparation from yeast efficiently releases autonomous plasmids for transformation of Escherichia coli. Gene 57: 267-272

Hofsli, E., Bakke, O., Nonstad, U. and Espevik, T. (1989) A flow cytometric and immunofluorescence microscopic study of tumor necrosis factor production and localization in human monocytes. Cell Immunol. 122: 405-415

Jeong, J.-Y. and Jue, D.-M. (1997) Chloroquine inhibits processing of tumor necrosis factor in lipopolysaccharide-stimulated RAW 264.7 macrophages. J. Immunol. 158: 4901-4907

Jue, D.-M., Sherry, B., Luedke, C., Manogue, K. R. and Cerami, A. (1990) Processing of newly synthesized cachectin/tumor necrosis factor in endotoxin-stimulated macrophages. Biochemistry 29: 8371-8377

Kilmartin, J. V. and Adams, A. E. (1984) Structural rearrangements of tubulin and actin during the cell cycle of the yeast Saccharomyces. J. Cell Biol. 98: 922-933 
Kim, K.-U., Kwon, O.-J. and Jue, D.-M. (1993) Pro-tumour necrosis factor cleavage enzyme in macrophage membrane/ particulate. Immunology 80: 134-139

Kriegler, M., Perez, C., DeFay, K., Albert, I. and Lu, S. D. (1988) A novel form of TNF/cachectin is a cell surface cytotoxic transmembrane protein: ramifications for the complex physiology of TNF. Cell 53: 45-53

Lunn, C. A., Fan, X., Dalie, B., Miller, K., Zavodny, P. J., Narula, S. K. and Lundell, D. (1997) Purification of ADAM 10 from bovine spleen as a TNFa convertase. FEBS Lett. 400: 333-335

McGeehan, G. M., Becherer, J. D., Bast, R. C. Jr., Boyer, C. M., Champion, B., Connolly, K. M., Conway, J. G., Furdon, P., Karp, S., Kidao, S., McElroy, A. B., Nichols, J., Pryzwansky, K. M., Schoenen, F., Sekut, L., Tuesdale, A., Verghese, M., Warner, J. and Ways, J. P. (1994) Regulation of tumour necrosis factor-a processing by a metalloproteinase inhibitor. Nature 370: 558-561

Merlos-Suarez, A., Fernandez-Larrea, J., Reddy, P., Baselga, J. and Arribas, J. (1998) Pro-tumor necrosis factor- $\alpha$ processing activity is tightly controlled by a component that does not affect notch processing. J. Biol. Chem. 273: 24955-24962

Mohler, K. M., Sleath, P. R., Fitzner, J. N., Cerretti, D. P., Alderson, M., Kerwar, S. S., Torrance, D. S., Otten-Evans, C., Greenstreet, T., Weerawarna, K., Kronheim, S. R., Petersen, M., Gerhart, M., Kozlosky, C. J., March, C. J. and Black, R. A. (1994) Protection against a lethal dose of endotoxin by an inhibitor of tumour necrosis factor processing. Nature 370: 218-220

Moss, M. L., Jin, S.-L. C., Milla, M. E., Brrkhart, W., Carter, H. L., Chen, W.-J., Hoffman, C. R., Kost, T. A., Lambert, M. H., Leesnitzer, M. A., McCauley, P., McGeehan, G., Mitchell, J., Moyer, M., Pahel, G., Rocque, W., Overton, L. K., Schoenen, F., Seaton, T., Su, J.-L., Warner, J., Willard, D. and Becherer, J. D. (1997) Cloning of disintegrin metalloproteinase that processes precursor tumour-necrosis factor- $\alpha$. Nature 385 : 733-736
Peschon, J. J., Slack, J. L., Reddy, P., Stocking, K. L., Sunnarborg, S. W., Lee, D. C., Russell, W. E., Castner, B. J., Johnson, R. S., Fitzner, J. N., Boyce, R. W., Nelson, N., Kozlosky, C. J., Wolfson, M. F., Rauch, C. T., Cerretti, D. P., Paxton, R. J., March, C. J. and Black, R. A. (1998) An essential role for ectodomain shedding in mammalian development. Science 282: 1281-1284

Rosendahl, M. S., Ko, S. C., Long, D. L., Brewer, M. T., Rosenzweig, B., Hedl, E., Anderson, L., Pyle, S. M., Moreland, J., Meyers, M. A., Kohno, T., Lyons, D. and Lichenstein, H. S. (1997) Identification and characterization of a pro-tumor necrosis factor- $\alpha$-processing enzyme from the ADAM family of zinc metalloproteases. J. Biol. Chem. 272: 24588-24593

Solomon, K. A., Pesti, N., Wu, G. and Newton, R. C. (1999) A dominant negative form of TNF- $\alpha$ converting enzyme inhibits proTNF and TNFRII secretion. J. Immunol. 163: 4105-4108

Tracey, K. J., Beutler, B., Lowry, S. F., Merryweather, J., Wolpe, S., Milsark, I. W., Hariri, R. J., Fahey, T. J. 3d., Zentella, A., Albert, J. D., Shires, G. T. and Cerami, A. (1986) Shock and tissue injury induced by recombinant human cachectin. Science 234: $470-474$

Tracey, K. J. and Cerami, A. (1993) Tumor necrosis factor, other cytokines and disease. Annu. Rev. Cell Biol. 9: 317-343

Vassalli, P. (1992) The pathophysiology of tumor necrosis factors. Annu. Rev. Immunol. 10: 411-452

Wallach, D., Varfolomeev, E. E., Malinin, N. L., Goltsev, Y. V., Kovalenko, A. V. and Boldin, M. P. (1999) Tumor necrosis factor receptor and Fas signaling mechanisms. Annu. Rev. Immunol. 17: 331-367

Werb, Z. and Yan, Y. (1998) A cellular striptease act. Science 282: $1279-1280$

Yang, J. M., Cho, C. H., Kong, K. A., Jang, J. S., Kim, H. W. and Juhnn, Y. S. (1999) Increased expression of Goq protein in the heart of streptozotocin-induced diabetic rats. Exp. Mol. Med. 31: 179-184. 